ENCYCLOPEDDIE Encyclopédie berbère

BERBERE

$34 \mid 2012$

34| Nemencha - Nybgenii

\title{
Nombres / Numération
}

Salem Chaker

\section{OpenEdition}

Journals

Édition électronique

URL : https://journals.openedition.org/encyclopedieberbere/2756

DOI : $10.4000 /$ encyclopedieberbere. 2756

ISSN : 2262-7197

\section{Éditeur}

Peeters Publishers

\section{Édition imprimée}

Date de publication : 5 octobre 2012

Pagination : 5610-5616

ISBN : 978-90-429-2718-6

ISSN : 1015-7344

\section{Référence électronique}

Salem Chaker, « Nombres / Numération », Encyclopédie berbère [En ligne], 34 | 2012, document N67, mis en ligne le 15 mars 2021, consulté le 17 février 2022. URL : http://journals.openedition.org/ encyclopedieberbere/2756 ; DOI : https://doi.org/10.4000/encyclopedieberbere.2756

Ce document a été généré automatiquement le 17 février 2022.

(C) Tous droits réservés 


\title{
Nombres / Numération
}

\author{
Salem Chaker
}

1 Les noms de nombres et le système de numération berbères n'ont fait l'objet que d'un nombre limités de travaux. La bibliographie sur le sujet est très ramassée et les références essentielles se ramènent à quelques auteurs, essentiellement: A. Basset (1952/1969), Klingenheben (1927), Galand (1967 et 1978), Prasse (1974), Zavadovskij (1974) et, plus récemment, dans le cadre d'une approche large dépassant la seule langue berbère : Blažek (1999), Alonso de Lafuente (2007).

\section{Le système de numération berbère}

2 Si les parlers berbères actuels présentent tous un système de numération décimal, un certain nombre d'indices généraux et de données locales indiquent qu'il a dû exister antérieurement un système de numération quinaire. R. Basset (1892, p. 27-28) et de Calassanti-Motylinski 1898, p. 31) ont signalé pour le Dj. Nefoussa*, Le Mzab*, Ouargla* et les parlers de l'oued Righ, l'utilisation du mot afus*, "main "* (ou ses variantes locales, ufes, fus) pour désigner le nombre 5 ; avec la possibilité, au-delà de 5 , de combiner le mot afus + un chiffre : afus d iggen, "main » et $1=6$, etc. (voir aussi, pour quelques précisions, Vycichl 1985). L'usage de fus, «main » pour 5 est également attesté en Libye à Sokna, dans un système qui mobilise aussi le "doigt ", dad pour l'unité et les «pieds » pour la vinfgtaine (cf. Prasse 1982). Une autre trace de l'existence d'un ancien système quinaire pourrait résider dans l'importance du symbole de la main (cf. notices A83 «Afus», C67 "Cinq" et M16 "Main») et de sa valeur numérique et organisationnelle en anthropologie sociale; en de nombreux points du monde berbère, les tribus et/ou confédérations sont organisées en cinq sous-composantes (Hart 1967), ce qui induit que les mots afus (« main ») ou tawsit (« paume ») puissent désigner aussi, notamment en tachelhit et en touareg, la tribu ou la confédération de tribus, en référence à la main ouverte d'où divergent (ou convergent) les cinq doigts de la main (ddukklən am idudan ufus, « ils sont unis comme les (cinq) doigts de la main », dit-on en kabyle). 
3 L'inventaire de formes (cf. infra) et le fait que les nombres berbères de 5 à 9 pourraient bien être un emprunt ancien au sémitique tendraient également à confirmer que le système de numération primitif ne comportait qu'une série de 1 à 5 , ce dernier chiffre étant représenté par afus, "main»; le système de représentation graphique des nombres à Ghadames va également dans le même sens (cf. infra). Cela permettrait aussi de comprendre pourquoi le nombre 10, moraw, ne semble pas avoir de correspondant chamito-sémitique : il s'agirait d'une forme lexicale initialement étrangère au système de numération proprement dit qui aurait été spécialisée dans une phase secondaire.

\section{L'inventaire des formes}

4 Actuellement, la plupart des dialectes ont emprunté à l'arabe (dialectal) les noms de nombre à partir de 3 (kabyle) ou 4 (tamazight). Seuls les dialectes " méridionaux » (touareg, chleuh, mozabite, zenaga) conservent la série complète des noms de nombre de 1 à 10. Dans cet ensemble, seul le touareg maintient un usage complet et généralisé de la numération berbère : dans les autres parlers, au-delà de 10 (parfois au-delà de 20 seulement), on passe généralement à la numération arabe.

Une des spécificités de ce paradigme berbère est l'opposition systématique de genre, masc./fem, en accord avec le genre du nom dénombré.

\begin{tabular}{|c|c|c|c|}
\hline & Kabyle & Touareg & Sémitique \\
\hline 1. & yiwə/yiwət & iyən/iyət & whd / we(d) (égyptien) \\
\hline 2. & sin/snat & əssīn/sənātət & $\underline{t} n$ \\
\hline 3. & *tlata & kəraḍ/kərāḍt t & tlt $\underline{t}$ \\
\hline 4. & *rebsa & okkoz/okkōẓət & $r b \varepsilon$ \\
\hline 5. & *xemsa & səmmus/səmmūsət & $x m s$ \\
\hline 6. & $*_{\text {setta }}$ & səḍis/səḍisət $t$ & šdš/sds \\
\hline 7. & *sebsa & əssa/əssāhət & $s b \varepsilon$ \\
\hline 8. & $*_{\text {tmanya }}$ & əttām/əttāmət & $\underline{t} m-n y$ \\
\hline 9. & $*_{t t \partial s \varepsilon a}$ & təža/təžzahət & $t \check{s} \varepsilon / t s \varepsilon$ \\
\hline 10. & 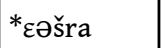 & məraw/mərawət & $\varepsilon s ̌ r$ \\
\hline 100. & *miyya & temede (Mzab : twinəst) & \\
\hline 1000. & *aləf & agim & \\
\hline
\end{tabular}

* EMPRUNTS À L'ARABE. 


\section{L'origine des formes}

6 L'étude systématique de Zavadovskij (1974), largement recoupée par Prasse (1974) ${ }^{1}$ établit des correspondances globalement satisfaisantes avec des formes chamitosémitiques pour la grande majorité des noms de nombre (8 sur 10); seuls semblent «résister» 4 et 10 . On nuancera cependant ces conclusions car, depuis longtemps, l'hypothèse d'un emprunt ancien a été envisagée tant les formes berbères sont proches de celles du sémitique ${ }^{2}$. En fait, l'examen de la série fait immédiatement ressortir une nette dissemblance entre la séquence de 1 à 4 et la séquence de 5 à 9 . Pour cette dernière, les ressemblances avec le sémitique sont tellement fortes et immédiates que l'on est en droit de suspecter un emprunt global au sémitique, sans doute au phénicien ou au punique ; alors que la première partie de la série (1 à 4) présente une spécificité berbère plus marquée, qui met en jeu des correspondances phonétiques plus complexes avec les données chamito-sémitiques. C'est d'ailleurs ce que semble envisager Zavadoskij (1974, p. 104) quand il écrit :

7 "L'indice de genre [-ət] a été dans certains cas ajouté en berbère non pas à des formes chamito-sémitiques complètes, mais à des racines sémitiques tronquées (par exemple Sa, TaM, TZa)».

8 Il n'est donc pas du tout impossible que le berbère ait emprunté deux fois les noms de nombres au sémitique : une première fois, les nombres de 5 à 9 , au phénico-punique ; une seconde fois, pour la majorité des dialectes, les nombres à partir de 3 (ou 4), à l'arabe.

9 La détermination socio-économique, dans les deux cas, serait évidemment la même : les échanges commerciaux avec des marchands de langue sémitique. C'est un phénomène universellement observé : lors des contacts et échanges commerciaux entre «peuples autochtones " et commerçants étrangers ayant un système socio-économique plus complexe, la numération de ces derniers s'impose comme outil de l'échange ${ }^{3}$.

\section{Une origine verbale probable}

Des traces nombreuses (en touareg) et assez largement distribuées (touareg, chaoui, kabyle) indiquent que les noms de nombres berbères ont connu des formes verbales et sont peut-être eux-mêmes d'anciennes formes verbales conjuguées (verbes d'état à la $3^{\mathrm{e}}$ pers. sing. masc./fém.). Les formes touarègues : əssənən, « tous les deux » (cf. əssin); əkkərdən, «tous les trois (cf. kərad) ; əkkəzən, « tous les quatre» (cf. okkoz), etc., qui ont été analysées par K.-G. Prasse (1974, p. 408) comme des " nombres collectifs », sont en fait probablement la trace d'une conjugaison suffixale (suffixe $-n$ de $3^{\mathrm{e}}$ p.m.p. $=$ «ils »): « ils sont deux, trois, quatre... ». Cette hypothèse est confirmée par certaines données chaoui et kabyles (isnin, «tous les deux»; cf. Basset 1946), et plus fondamentalement par la morphologie du nombre 1 (yiwən/yiwət, « un/une ») qui ne peuvent guère s'expliquer autrement que par une origine verbale (suffixe de participe masc./fém. sur verbe d'état). 


\section{La syntaxe du nom de nombre}

11 La syntaxe du nom de nombre a été précisément étudiée par L. Galand (1967 et 1974) qui fournit une bonne synthèse sur le sujet. Contrairement à bien d'autres langues, le nom de nombre berbère n'est pas un déterminant mais un nominal déterminé par le nom qu'il dénombre (cf. N62, « Nom »); dans la suite :

\begin{tabular}{|l|l|l|}
\hline Nombre & $\leftarrow$ & Nom \\
\hline yiwən & & wərgaz \\
\hline un & & homme (+ E.A.) \\
\hline
\end{tabular}

c'est le nombre «un » qui est le déterminé, et le nom subséquent le déterminant (la subordination étant marquée par l'état d'annexion*).

13 En règle générale, de 1 à 9 , le syntagme " Nombre $\leftarrow$ Nom déterminant " est marqué uniquement par l'état d'annexion sur le nominal dénombré. A partir de 10, on a en principe un syntagme déterminatif (complément de nom) classique à préposition $n$ («de $»)$ :

məraw $\boldsymbol{n}$ təmyarin

dix de vieilles $=$ « Dix vieilles »

L. Galand a opposé nettement les deux constructions; dans la première, le nom dénombré étant considéré comme un "complément explicatif» (cf. notice F35, «Fonctions »), dans la seconde comme un complément déterminatif. Cette analyse renvoie à la conception qu'il a souvent développée quant à la spécificité de la fonction « complément explicatif». C'est sans doute accorder une grande importance à des faits de morphologie (présence/absence de la préposition n), qui paraissent syntaxiquement assez superficiels et de toute façon secondaires. L'essentiel au plan de la syntaxe est, dans les deux cas, le statut de complément du nom dénombré.

Plusieurs parlers marocains du dialecte tamazight du Moyen Atlas (cf. notamment Galand 1967, p. 257-258) utilisent le morphème de prédication nominale $d$ comme outil de liaison entre le nombre et le nominal :

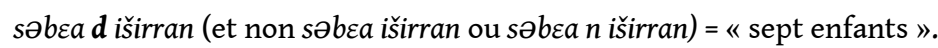

16 Ces constructions ont certainement influencé l'arabe dialectal marocain qui présente des séquences assez similaires. On notera que l'usage de ce morphème prédicatif $d$ dans des contextes syntaxiques de type déterminatifs n'a rien d'exceptionnel : on en trouve de nombreuses autres traces, post-nominales et post-verbales.

Enfin, partout, on constate une forte tendance des noms de nombres à fonctionner comme des «pronoms» (Galand 1978) et à avoir ainsi une double fonction, celle de noms de nombres et celle de substituts indéfinis. Cela est particulièrement sensible pour " un/une » : un énoncé kabyle, tout à fait banal en début de récit, comme : yəlla уішәП..., il existait un..., signifie : « il y avait quelqu'un/il y avait un homme... »; de même, son correspond chleuh yan devient quasiment un pronom indéfini comparable $\mathrm{au}$ « on » du français. 


\section{Les nombres ordinaux}

18 «Premier » et « dernier » sont généralement des dérivé nominaux (noms d'agent) du verbes zwir "précéder» (> aməzwaru, aməzwar, celui qui précède $=$ premier) et gguri " être en dernier, être en arrière " (anəggaru, celui qui est en dernier = dernier). Le touareg utilise une autre base lexicale (əlkəm, «suivre, venir après »: wa yəlkəmən). Les autres ordinaux sont construits sur des syntagmes du type «support de détermination " (pronom démonstratif défini ou indéfini) + nombre, selon deux modalités principales :

$19-\boldsymbol{w i} / \mathbf{t i} \boldsymbol{s}($ ou $\boldsymbol{w a} / \mathbf{t a} \boldsymbol{s})+$ nombre = celui/celle avec X, de loin la plus répandue (kabyle, chaoui, chleuh, tamazight...) qui doit être interprété comme « celui/celle avec lequel/ laquelle X (est complet) », wi-s-sin, « le second », ti-s-snat, « la seconde »,

$20 \mathrm{ou}$

$21-\boldsymbol{w a} n+$ nombre = celui de X $(\mathrm{X}=2,3 \ldots)$, que l'on relève essentiellement en touareg, mais aussi à Ouargla, semble secondaire (Prasse 1974, p. 407).

\section{Un cas de représentation graphique des nombres: Ghadames}

Un système spécifique de représentation des nombres a été signalé à Ghadames dès 1860 (Reinaud/Hanoteau), confirmé par Calassanti-Motylinski (1904) puis Lanfry 1968. Il est possible qu'il soit lié au statut de plaque tournante du commerce caravanier de Ghadames et/ou au caractère crucial du problème de la gestion des eaux d'irrigation qui a nécessité une forme d'enregistrement graphique; il n'est donc pas exclu qu'il existe/ait existé ailleurs en région saharienne ou présaharienne, le même système ou un système comparable. Si l'on considère l'ensemble des chiffres y compris ceux correspondant aux grands nombres, ses formes, sont assez hétérogènes : on y reconnaît des signes qui pourraient être des lettres de l'alphabet arabe $(50=\tau)$ ou de l'alphabet tifinagh $(X=500)$, voire libyque $(=1000)$; mais si l'on se focalise sur la série des dix premiers chiffres, le système présente une forte analogie avec les chiffres romains, dont il pourrait être une réminiscence lointaine comme le pensait Hanoteau : « ce n'est peut-être qu'un souvenir confus des chiffres romains qui offrent des combinaisons analogues » (Reinaud/Hanoteau 1860, p. 53). Werner Vycichl, pour sa part, évoque dans son article de 1952 une origine sud-arabique, en formulant l'hypothèse d'une transmission par les premiers conquérants arabes (p. 83). On restera dubitatif devant cette théorie qui est à la fois bien complexe et peu convaincante au plan des formes, en dehors peut-être du cas de $\bigcirc(=10)$, dans lequel on peut aussi voir le $\bigcirc$ tifinagh (= « $\mathrm{R} »)$ de Təraw ([○ :), « dix ». L'origine romaine - mâtinée d'autres influences locales : tifinagh, arabe - paraît infiniment plus simple et plus plausible, historiquement et géographiquement. On peut aussi penser qu'il s'agit d'une représentation analogique des doigts de la main et de la main elle-même (afus); ce système pourrait constituer alors un autre indice de l'existence d'un système de numération berbère quinaire puisqu'au-delà de 5 , la représentation des nombres est composée du chiffre $5+$ le nombre d'unités nécessaires $(6=1+5,7=2+5 \ldots)$. On imagine aisément la personne tenant horizontalement sa main droite ouverte et indiquant le nombre d'unités complémentaires de sa main gauche. 


\begin{tabular}{|l|l|}
\hline 1. & $\mid$ \\
\hline 2. & $\|$ \\
\hline 3. & $|\||$ \\
\hline 4. & $\mid\|\|$ \\
\hline 5. & $>$ \\
\hline 6. & $\mid>$ \\
\hline 7. & $\|>$ \\
\hline 8. & $|\||>$ \\
\hline 9. & $|\||>$ \\
\hline 10. & 0 \\
\hline
\end{tabular}

[Le système de représentation des chiffres de 1 à 10 à Ghadames ; d'après J. Lanfry 1968, p. 378].

\section{BIBLIOGRAPHIE}

ALONSO DE LAFUENTE J. A., 2007 - « Notas sobre la etimologia de los numerales beeberes », Anaquel de Estudios Árabes, 18, p. 41-63.

BASSET A., 1946 - « Berbère isnin, « tous les deux » ", CR du GLECS, IV, p. 19-20.

BASSET A., 1952 (1969) - La langue berbère, London, IAI [p. 28-29].

BASSET R., 1892 - Etude sur la zenatia du Mzab, de Ouargla et de l'oued Rir', Paris, Leroux.

BLAŽEK V., 1999 - Numerals. Comparative-etymological analysis of numeral Systems and their implications, (Saharan, Nubian, Egyptian, Berber, Kartvelian, Uralic, Altaic and Indo-European languages), Brno, Spisy Masarikovy University.

BRUGNATELLI V., 1981 - « Osservazioni preliminari sulla rilevanza dei numerali nella comparazione camito-semito/indeuropea ", Atti del Sodalizio Glottologico Milanese 21, p. 88-93.

CABRERA BARETTO M., 1971 - « Die Zahlwörter der Altkanarier », Almogaren, II, p. 151-166.

CALASSANTI-MOTYLINSKI A. (de), 1898 - Le Djebel Nefoussa, Paris, Leroux.

CALASSANTI-MOTYLINSKI A. (DE), 1904 - Le dialecte berbère de Ghadamès, Paris, Leroux [p. 253].

CAMPS G., 1994 - « Cinq (semmes/semmus) », Encyclopédie berbère, XIII, Aix-en-Provence, Edisud, p. $1958-1960$. 
CORTADE J.-M., 1969 - Essai de grammaire touarègue, Alger, IRS.

GALAND L., 1967 - « La construction du nom de nombre dans les parlers berberbères», Verhandlungen des zweiten Internationalen Dialektologenkongresses, I, (Zeitschrift für Mundartforhcung), Wiesbaden, Franz Steiner Verlag, p. 253-259.

GALAND L., 1978 - « La double fonction du nom de nombre », Atti del secondo congresso internationale di linguistica camito-semitica (Firenze, 1974), Università di Firenze (Quaderni di Semistica : 5), p. 301-305.

HART D. M., 1967 - "Segmentary System and the role of the « five fifth » in tribal Morrocco", Revue de l'Occident Musulman et de la Méditerranée, 3, p. 65-95.

KLINGENHEBEN A., 1927 - “Zu den Zahlmethoden in den Berbersprachen”, Zeitschrift für Eingeborenen Sprachen, XVII, p. 40-51.

LANFRY J., 1968 - Ghadames.. I. Textes..., Fort-National, FDB [p. 378].

PRASSE K.-G., 1974 - Manuel de grammaire touarègue (Tahaggart), IV-V Nom, Copenhague, Akademisk Forlag [p. 403-408].

REINAUD M., 1860 - Notices sur les dictionnaires géographiques arabes et sur le système primitif de numeration chez les peuples de race berbère, (extrait du Journal asiatique), p. 50-54 [lettre du 29 octobre 1860 de A. Hanoteau].

RÖSSLER O., 1966 - "Das ältere Umschreibungssystem für Fremdnamen und seine sprachwissenschaftlichen Lehren”, Neue Afrikanistische Studien (Hamburg), 5, p. 218-219.

VYCICHL W., 1952 - « Das berberische Zyffernsystem von Ghadames und seine Ursprung », Rivista degli Studi Orientali, XXVII, p. 81-83.

VYCICHL W., 1985 - "Das Zeichen für d « Hand » in der Hieroglyphenschrift und die semitische Entsprechungen des zugrunde liegenden Etymons", [Le signe pour $d$ « main » dans l'écriture hiéroglyphique et les expressions sémitiques de l'étymon sous-jacent], Zeitschrift für ägyptische Sprache und Altertumkunde, 112, p. 169-179.

WÖLFEL D. J., 1954 - « Les noms de nombre dans le parler guanche des îles Canaries», Hespéris, XLI, p. 47-79.

ZAVADOVSKIJ J. N., 1974 - « Les noms de nombre berbères à la lumière des études comparées chamito-sémitique ", Actes du premier congrès international de linguistique sémitique et chamitosémitique (Paris, 1969), La Haye/Paris, Mouton, p. 103-111.

\section{NOTES}

1. Sur l'analyse étymologique des formes, on se reportera également à Blažek (1999) qui aborde le sujet dans un cadre élargi à de nombreuses autres familles linguistiques et au commentaire ciblé qu'en propose Alonso de Lafuente (2007).

2. Par ex., A. Basset 1952, p. 28 : «L'ancienne série qui n'est pas nécessairement berbère d'origine... ». Cf. également Prasse 1974 qui relie directement certains des noms de nombres à des formes sémitiques (même si ses formulations ne sont pas explicites quant à la thèse de l'emprunt). Luigi Serra pour sa part a évoqué expressément l'hypothèse d'un emprunt au sémitique de la série des nombres de 5 à 9 (cf. Brugnatelli 1981, note 3).

3. Ainsi, les Inuits du Canada comptent désormais en anglais même lorsqu'ils utilisent leur langue. 
INDEX

Mots-clés : Linguistique 